\title{
Technological Developments in Neonatal Care at Birth
}

David J R Hutchon*

Emeritus Consultant Obstetrician, Memorial Hospital, Darlington, United Kingdom

*Corresponding author: David J R Hutchon, Emeritus Consultant Obstetrician, Memorial Hospital, Darlington, United Kingdom, Tel: 815.753.6584; E-mail: djrhutchon@hotmail.co.uk

Received date: August 25, 2014, Accepted date: November 19, 2014, Publication date: November 24, 2014

Copyright: (c) 2014 Hutchon DJR. This is an open-access article distributed under the terms of the Creative Commons Attribution License, which permits unrestricted use, distribution, and reproduction in any medium, provided the original author and source are credited.

\begin{abstract}
During labour the fetal heart rate is the primary measurement to determine the health of the fetus and full use of modern electronics is made to measure the rate and variability in the rate which occurs in both a healthy and compromised fetus. Real time documentation is also part of the process. At birth the heart rate remains the primary vital sign of the neonate but measurement now relies on auscultation without any real-time documentation, at least until a satisfactory signal from pulse oximetry can be obtained. Pulse oximetry takes at least one minute before the signal is reliable but during this first minute critical decisions regarding care have to be made and during this time critical damage to the neonatal brain can occur if the wrong decision has been made. We describe how this gap in accurate measurement and documentation can be closed.
\end{abstract}

Keywords: Midwife; Nursing; Neonatal heart rate

\section{Introduction}

The neonatal 2010 International Liaison Committee on Resuscitation (ILCOR) recommendation was for a delay of at least one minute before clamping the umbilical cord of the neonate at birth. However for the baby in need of ventilatory support the committee stated that positive pressure ventilation was the priority and evidence is insufficient to recommend a time for clamping in those who require resuscitation. They also stated that the primary vital sign to judge the need for resuscitation should remain the heart rate and the heart rate should also provide evidence of the efficacy of the resuscitation. Assessment of the heart rate should be by auscultation of the precordium. The committee considered that there is a high likelihood of underestimating the heart rate with palpation of the umbilical pulse, but this is preferable to other palpation locations [1].

In the ILCOR algorithm the first measurement of the heart rate should take place by 30 seconds after birth. Measurement of a heart rate is provided in beats per minute and while counting for a whole minute is not always required, the heart rate in beats per minute does require auscultation over an accurately measured and sufficient length of time. In practice in a noisy delivery room the accuracy of the measurement must be compromised both in terms of actual counting of the heart beats and of the accuracy of the interval over which the beats are counted. This accuracy contrasts with the cardiotocograph (CTG) used during labour. Furthermore measurement of the heart rate by auscultation at birth is undocumented in real-time and not available for subsequent audit. Documentation is almost completely obscured within the aggregated APGAR score [2].

The ILCOR committees have recommended that oximetry be used to measure and document the neonatal heart rate and this is potentially as accurate and documentable as the CTG was during labour for the fetal heart rate. However oximetry does not provide an output during the first minute after birth when the most critical decisions on resuscitation need to be made [3].
Without accuracy and documentation of the neonatal heart rate at birth which drives the critical decision on whether or not resuscitation should be initiated, review and audit for the purpose of advancing neonatal resuscitation and care at birth is not possible [4]. Since there is logical and clinical evidence that maintaining the placental circulation for a few minutes after birth benefits the term and preterm infant, it seems logical that maintaining the placental circulation will also benefit the compromised neonate who requires resuscitation [5-7]. Currently, using the ILCOR algorithm, if the heart rate is underestimated, the neonate may be subjected to early cord clamping which will effect changes in the circulation [6] and may then precipitate a requirement for ventilatory support which may not have otherwise been needed.

\section{Technological solution}

Determining the heart rate was one of the earliest uses of electronic technology in recent times, with the CTG and oximetry as prime examples. Cardiotocography can determine the fetal heart rate using either Doppler ultrasound or the fetal ECG signal. During pregnancy doppler ultrasound is routinely used to detect and measure the fetal heart rate and has become both reliable and low cost technology. It seems logical to continue to use the doppler ultrasound immediately after birth, until at least a reliable oximetry signal can be obtained.

\section{Method}

Alternative approaches to using Doppler ultrasound to monitor the neonatal heart rate were considered and a literature search carried out. Long before ultrasound Doppler technology was available the neonatal heart rate could be measured with the neonatal ECG. (Brady et al). This has also been used recently in research. The ECG can be considered the gold standard but in practice with current technology it may not be easy to get the electrodes attached to the wet newborn infant in a clinical situation, and muscle artefact signals may interfere with the signal. (Personal communication). No other alternative solutions were found. 
Page 2 of 3

\section{Results}

With the proximity of the heart to the ultrasound probe we considered that the signal would be easily obtained and this provides to be the case. The author has shown that an ultrasound heart rate is readily obtained immediately after birth in the newborn piglet weighing about $1 \mathrm{~kg}$ (Figure 1), equivalent to a preterm neonate. This approach was confirmed to be equally effective in humans in a recent study [8]. This study also confirmed that the time taken for a reliable precordial Doppler ultrasound signal was within a few seconds of birth, and significantly faster than the heart rate determined by precordial auscultation. In the preterm baby, placed inside a polythene bag to help maintain its temperature, ultrasound will work well through the polythene whereas getting an ECG signal may involve exposing the neonate outside the bag with an increased risk of hypothermia.

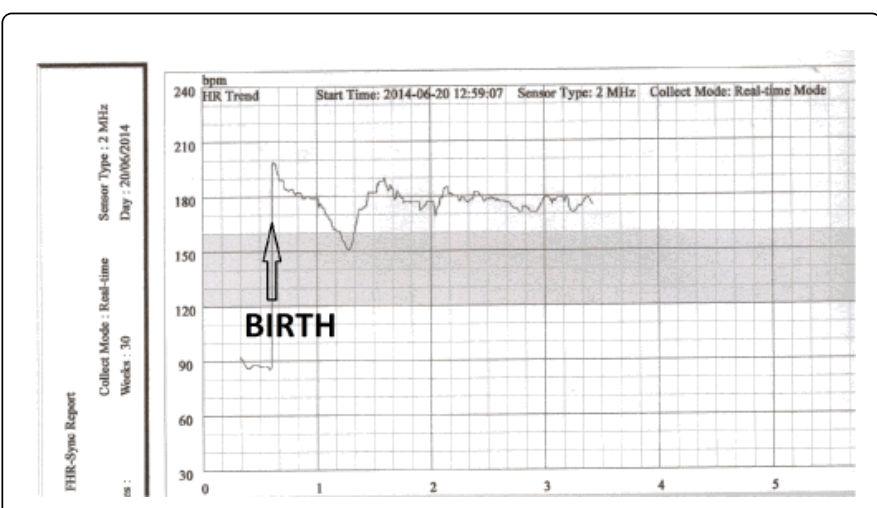

Figure 1: Heart rate of newborn piglet using precordial ultrasound Doppler machine with recording facility.

\section{Discussion}

\section{Research and Audit}

As explained above, without an accurate and documented record of the neonatal heart rate at birth, combined with an accurate and documented fetal heart rate during labour, it is very difficult to determine where improvements in perinatal care are possible. Until recently the impact of early cord clamping on the condition of the neonate was largely unrecognised and as a result the timing of the intervention was neither measured not documented. Although it is likely that early clamping was preformed in the majority of hospital births [9], even in hospital births there is likely to be some variability in the timing, and a brief delay may have a significant impact on neonatal outcome [10]. Currently the neonatal heart rate is not documented as an actual rate, but scored as "absent" or "present" and/or above or below 100 beats per minute within the APGAR score. When the inaccuracy of the measurement of the heart rate is taken into account it can be appreciated that such documentation is much too crude to expect to be able to assess the value of different clinical approaches of cord clamping.

\section{Current Neonatal Resuscitation}

There are numerous neonatal resuscitaires available commercially and all are designed to be used at the side of the room rather than close enough to the mother to permit the umbilical cord to remain intact.
The acceptance of room-side resuscitaires was, as explained above, because the role of the placental circulation during the first minute or so after birth was not understood. The room-side resuscitaire is limited in mobility, height and mother-side friendly features. Adaption of the current equipment is far from straight forward. While adaptation is possible, it is clumsy and inconvenient [11] and a compromise which is not necessary with modern technological advances. A completely new design approach was required which provides a stable surface for the neonate, accessible to the attendants to be able to provide all the currently proven facilities for maintaining temperature, providing ventilation, suction and oxygen supplementation and monitoring $[4,12]$.

Room-side resuscitation has become a standard of care which is accepted by mother who was unaware of any alternative. However the distress caused by such separation at the moment of birth can only be imagined and an attempt has only recently been made to assess the impact of this separation on the mother [13]. A neonate that has been accustomed to the sound and smell of its mother and suddenly subjected to alien sounds and smells can only become further distressed [14]. The calming effect of the mother's touch leading to improved temperature control, higher breast feeding rates and the soothing effect of immediate skin to skin contact of the neonate at birth are proven [15].

\section{Conclusion}

Accurate measurement of the neonatal heart rate with Doppler ultrasound is possible and can easily be seen as an extension of the fetal heart rate monitoring and documentation. The approach presents a minor technological development challenge. Redesign of the resuscitation trolley to allow ventilation of the neonate at the mother's side with the cord still intact has been completed [4] and evaluation of the approach is underway. This approach will require the cooperation of nursing, obstetrical and neonatal staff, and will present a particular challenge for co-operation in babies' delivered by caesarean section and assisted vaginal delivery. However with fewer babies requiring resuscitation as a result of a policy of delayed cord clamping [16], the spared nursing resources can be redirected to providing increased attention to the measurement of the neonatal heart at birth. High risk babies will already be monitored by the CTG and a simple modification is needed to provide a transducer suitable for the neonatal precordium and allow a continuous record of the heart rate during birth.

The estimated cost of early cord clamping has been estimated to be around one billion euros [17] and only a small fraction of this is needed to provide the technology, the equipment and the training to provide resuscitation with the cord intact and routine delayed cord clamping.

\section{References}

1. Wyllie J, Perlman M, Kattwinkel J,Atkins D,ChameidesL, et al. on behalf of the Neonatal Resuscitation Chapter Collaborators Neonatal Resuscitation. 2010. International consensus on cardiopulmonary resuscitation and emergency cardiovascular care science with treatment recommendations.

2. Clark DA, Hakanson DO (1988) The inaccuracy of Apgar scoring. J Perinatol 8: 203-205.

3. Dawson JA, Kamlin CO, Wong C, te Pas AB, Vento M, et al. (2010) Changes in heart rate in the first minutes after birth. Arch Dis Child Fetal Neonatal Ed 95: F177-181. 
Page 3 of 3

4. Hutchon D (2014) Evolution of neonatal resuscitation with intact placental circulation. Infant 2014; 10: 58-61.

5. Raju TN (2013) Timing of umbilical cord clamping after birth for optimizing placental transfusion. CurrOpinPediatr 25: 180-187.

6. Bhatt S, Alison BJ,Wallace EM, Crossley KJ, Gill AW, et al. (2013) Delaying cord clamping until ventilation onset improves cardiovascular function at birth in preterm lambs. J Physiol. 591: 2113-26.

7. Uwins C, Hutchon DJR (2014)Delayed umbilical cord clamping after child birth: potential benefits to baby's health.Pediatric Health, Medicine and Therapeutics.

8. Goenka S,Khan M,Koppel RI,Heiman HS (2014)Precordial Doppler Ultrasound Achieves Earlier and More Accurate Newborn Heart Rates in the Delivery Room. Poster 3843.590Pediatric Academic Societies and Asian Society for Pediatric Research.

9. Ononeze AB, Hutchon DJ (2009) Attitude of obstetricians towards delayed cord clamping: a questionnaire-based study. J ObstetGynaecol 29: 223-224.

10. World Health Organization (WHO) (2012) Guidelines on basic newborn resuscitation. Geneva:World Health Organization.
11. Hutchon DJR Thakur I (2007) How to resuscitate the neonate with the cord intact at caesarean section. London: British International Congress of Obstetrics and Gynaecology. 93: 451.

12. Weeks AD, Watt RJP, Hutchon DJR, Yoxall CW, Gallagher A (2005) Innovation in immediate neonatal care: development of the Bedside Assessment, Stabilisation and Initial Cardiorespiratory Support (BASICS) Trolley.

13. Arnold L, Sawyer A, Rabe H, Abbott J, Gyte G, et al. (2013) Parents' first moments with their very preterm babies: a qualitative study. BMJ Open 3.

14. Porter RH (2004) The biological significance of skin-to-skin contact and maternal odours. ActaPaediatr 93: 1560-1562.

15. Moore ER, Anderson GC, Bergman N (2007) Early skin-to-skin contact for mothers and their healthy newborn infants. Cochrane Database of Systematic Reviews.

16. Hutchon DJR. Early versus delayed cord clamping at birth; in sicknessand in health. Fetal and Maternal Medicine Review

17. Hutchon DJR, Wepster B(2014) The estimated cost of early cord clamping at birth within Europe. International Journal of Childbirth 